\title{
GPS Vehicle Positioning and Real-time Image System Design
}

\author{
Jingchao Liu ${ }^{1 *}$, Chunyan Feng ${ }^{2}$ \\ (Xijing University)
}

${ }^{1}$ Xijing University, No.1 Xijing Road, Chang'an District, Xi'an City, Shaanxi Province, China

${ }^{2}$ Xijing University, No.1 Xijing Road, Chang'an District, Xi'an City, Shaanxi Province, China

a20100058@xijing.edu.cn, ${ }^{b} 704032392 @ q q . c o m$

Keywords: GPS Positioning; Real-time Image; Car Theft Prevention

\begin{abstract}
In this paper, a design scheme of GPS vehicle positioning and real-time image system is introduced. Through effective integration of GPS positioning module, real-time image module and GPRS module, auto positioning, voice calling, image display and other functions are realized. It can also realize the detection and display function of alcohol, temperature and PM2.5 through expansion, which is very consistent with the needs of car owners and market demands.
\end{abstract}

\section{Introduction}

Since 1964, when the GPS system was officially put into use, the technology and the precision have been continuously improved. In 1994, the global coverage rate reached 98\%, with a total of 24 GPS satellites. Nowadays, civilian GPS detection technology can be accurate to within 10 meters and go deep into various fields of economic development. It has become a very important technology in automobile positioning, track tracking, system management and Internet application. Among them, the market share of vehicle navigation and positioning is the largest, and the use of anti-theft devices is also developing rapidly.

The increase in the number of cars has brought the congestion and difficulties in parking, and the camera-assisted reversing system has gradually become an essential part of the car. Because the camera assistant can provide more accurate and clear image display for car owners, it is also applied in the security system.

\section{Overall system design}

The design scheme of ARM based embedded system is based on the above market demand. It can realize a series of related functions, such as automobile positioning, message sending, voice communication and reversing image.

The Design Consists of Two Parts: Software and Hardware. The hardware part mainly guarantees the realization of GPS positioning, real-time camera shooting and cellphone signal reception. COMS image sensor is selected by the system. It has low cost and power consumption and is widely used in on-board camera.

The Software Part Controls the Reception, Identification, Coding, Transmission, Execution and Other Specific Operations. It mainly completes three functions. First, it completes the initialization of each part of the system and the circular processing after the GPS module receives the information. Secondly, communication between DSP controller and mobile phone is completed, and communication between vehicle owners and vehicles is realized. The third is to deal with other set abnormal conditions.

Through the Above Design, the System Can Achieve the Following Functions. (1) automobile GPS real-time positioning and recording; (2) reverse image display; (3) display and record driving images; (4) send the location information or image information to the designated mobile phone as required by the user or in case of an emergency. 


\section{System module design}

The system is composed of GPS positioning module (design board 1) and real-time image module (design board 2). GPS positioning module integrates voice module and GPRS module. The real-time image module consists of camera, transmission module and display. As shown in figure 1.

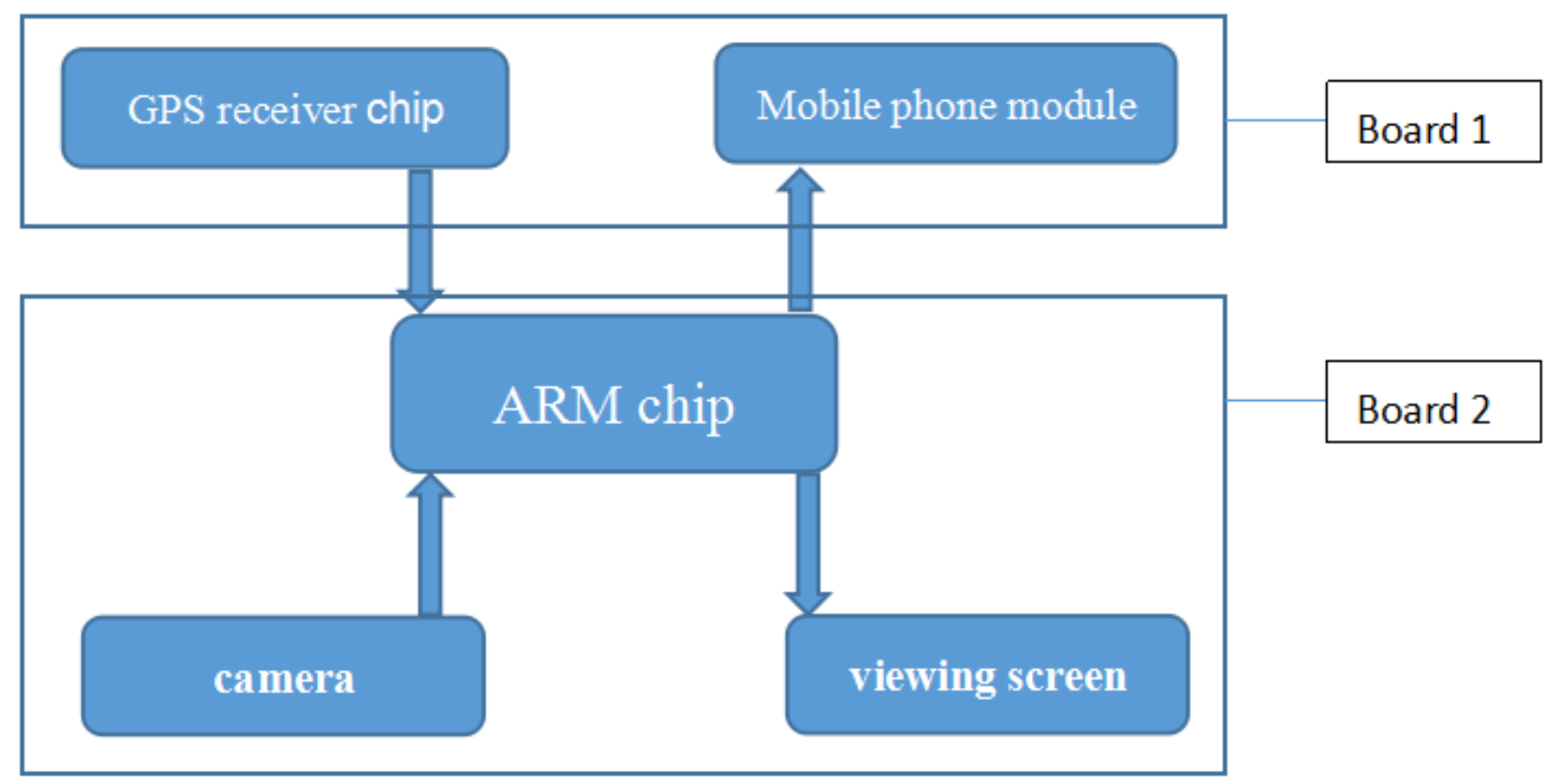

Figure 1. block diagram of GPS vehicle positioning and real-time image system design

The GPS Positioning Module. GPS positioning module consists of GPS receiver chip, mobile phone module, self-designed power supply, radio frequency antenna, speech module, recording module and other components.

The GPS receiver chip completes the reception of the GPS location signal and stores the information in the SD card, which is convenient for the user to query. The integrated view module and speech module can realize the function of the phone, and when you plug in the phone, you can not only answer the call, but also can communicate with the driver in real time to realize the remote control of the vehicle scheduling. At the same time, it can complete text messages and send the required information to the specified phone according to the settings program and user.

The Real-time Image Module. The real-time image module is composed of a camera, a transmission module and a display, in which the camera selects 300,000 pixels and the image resolution is $320 * 240$. A 2.8 -inch display is selected to match it.

After the effective integration of all modules, the real-time image module can collect the required image information as required by the user and transmit it to the system display device, or in case of emergency, trigger the recording switch and start recording the image information. Images captured by GPS data and cameras are stored on SD CARDS, which can be queried on a computer or be sent in a message to a designated phone. The system can also set several emergency switches in the car, which will be triggered in the event of an emergency, such as stolen. When the emergency switch is triggered, the system will automatically extract the GPS location information and real-time image information from the SD card according to the setting procedure and send it to the designated mobile phone. It provides effective information for the recovery of vehicles, the arrest of suspects and the restoration of the situation at that time.

\section{Design innovation and expansion}

This design combines GPS positioning, mobile phone short message, call and real-time image to 
achieve a variety of functions. Besides, the design reserved slot and expansion space, which can increase the temperature, PM2.5 test and display and other functions. In this way, the function of a nanny car is basically realized, which can be applied in various fields such as burglar alarm of the family car, real-time tracking of logistics company, safety control of reversing car, and unified control of subordinate car by the head office.

As the main component of haze, PM2.5 has attracted more and more attention. However, the traditional PM2.5 environmental detector is expensive, inconvenient to use and difficult to be used in families. Based on this, the intelligent vehicle-mounted PM2.5 environmental detector was designed based on sensor technology and STM32. It can use sharp GP2Y1010 sensor to complete the collection of dust particles, use temperature and humidity sensor DHT11 to complete the acquisition of indoor temperature and humidity. The information can be transformed by $\mathrm{AD}$, then put the result into the single chip microcomputer STM32 which controls the processing, and displays the information on the OLED screen. At the same time, you can set the transfinite alarm value, when the concentration of PM2.5 more than the set value, the sound light alarm and GSM module that send SMS alerts will work.

In order to effectively avoided accidents that due to driver's drunk driving, the design can contain a breathalyzer. It can use ARM series micro-controller as core controller, and use high sensitive electrochemical sensor, MQ-3 sensor, semiconductor alcohol sensor to complete the data collection. It makes the multi-sensor signals from the alcohol detection system together by the fuzzy control algorithm. The system can provide a variety of functions such as detection, display, alarm, control and recording, with the features of low cost, small volume and strong intelligence. It is known to have high accuracy through simulation.

\section{Summary}

In recent years, with the rapid development of national economy and the rapid improvement in people's living standard, the Chinese car industry has developed rapidly, and it will make the auto electronics industry stride forward. Now, people need the more characteristics such as safety of the car, comfortable, and multifunctional, so pure vehicle electronic equipment cannot meet the needs of the people. The introduction of automatic control technology, automotive electronics technology, multimedia technology, Internet technology, wireless communication and other technologies will make the design of a systematic, multifunctional vehicle-mounted information system become the future trend of vehicle-mounted equipment development. Therefore, it has become the main direction of the development on the automobile industry to ensure the safety and efficiency while pursuing the comfort, convenience and speediness, and to meet the needs of users in various aspects.

To sum up, this design can realize GPS positioning inquiry, GPS positioning SMS notification, real-time image display, telephone answering, voice scheduling, recording or monitoring. It can also be expanded to carry out alcohol, temperature, PM2.5 test and real-time display, which is worthy of the name of a nanny car. This design is cheap, compact and portable, which provide great convenience for car owner, and accord with the market demand.

\section{Reference}

[1] Hongzhu Cai, Jingchao Liu, Xu Wang, Qiang Liu. Design of GPS vehicle positioning query and real-time image system [J]. Henan science \& technology,2014(21):93.

[2] Jianpo Li, Yan Xie, Haitao Zhao, Xiansheng Zhang. Intelligent positioning system of ev charging station based on GPS [J]. Electric measurement and instrumentation,2013,50(11):119-122.

[3] Yang Gao. Research on the key technology of measured image real scene navigation [D]. Chang 'an university,2013.

[4] Wenjuan Li. Research on vehicle positioning and optimization technology in Internet of vehicles [D]. Nanjing university of posts and telecommunications, 2013. 
[5] Shuilong Zou, Jie Gao. Research on automobile GPS positioning anti-theft system based on Internet of things [J]. Jiangxi science,2012,30(06):822-827.

[6] Yanqing Liu. Automotive communication positioning technology of Internet of things [D]. Xihua university,2012.

[7] Haitao Lu, Tongpu Hou, Jian Li. Development of embedded automobile positioning system on-board terminal based on GPS and GPRS [J]. Computer and digital engineering,2010,38(06):150-153+174.

[8] Yu Jing, Zhenjun Du. Research on GPS vehicle positioning and anti-theft system based on GPRS short message [J]. Computer engineering and design,2007(17):4315-4318.

[9] Fengzhen $\mathrm{Hu}$. Research and application of automotive GPS satellite navigation global positioning system technology [J]. Petroleum instruments,2007(04):46-48+51+109.

[10] Zhe $\mathrm{Xu}$, Lin Cong. Application of global positioning system (GPS) in automobile transportation [J]. Shanxi transportation technology,2006(02):76-78. 